\title{
Pattern formation in a metastable, gradient-driven sandpile
}

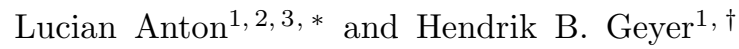 \\ ${ }^{1}$ Institute of Theoretical Physics, University of Stellenbosch, Private Bag X1, 7602 Matieland, South Africa \\ ${ }^{2}$ Department of Physics and Astronomy, University of Manchester, M13 9PL, U.K. \\ ${ }^{3}$ Institute of Atomic Physics, INFLPR, Lab 22, PO Box MG-36 R76900, Bucharest, Romania
}

(Dated:)

\begin{abstract}
With a toppling rule which generates metastable sites, we explore the properties of a gradientdriven sandpile that is minimally perturbed at one boundary. In two dimensions we find that the transport of grains takes place along deep valleys, generating a set of patterns as the system approaches the stationary state. We use two versions of the toppling rule to analyze the time behavior and the geometric properties of clusters of valleys, also discussing the relation between this model and the general properties of models displaying self-organized criticality.
\end{abstract}

PACS numbers: 82.40.Ck, 89.75.Da, 89.75.Fb

\section{INTRODUCTION}

Sandpile models were first introduced by Bak, Tang, and Wiesenfeld as explicit models of self-organized criticality (SOC) 1]. Since then a vast literature has analyzed sandpile properties resulting from various definitions of the toppling rules; see Refs. [2, 3, 4] for recent reviews.

In this paper we present the relaxation and stationary properties of a gradient-driven sandpile model with a metastable toppling rule. Interest in this type of work originates from seismology, where quasiperiodic behavior of seismic activity has been observed for certain faults and investigated with SOC-related models $[\underline{5}$. The model we present can describe characteristics of systems undergoing rheologic flow, e.g., in the mining environment or tectonic plates. In such systems stress can accumulate in various parts of the system for long periods of time, in contrast to normal fluid flow in which the local relaxation time is much smaller than the hydrodynamic time scale.

Furthermore, we believe that the properties we describe are of wider interest to other fields of nonequilibrium statistical mechanics where, e.g., stripe patterns similar to those that we observe appear in a variety of extended systems, including sand and biological systems [, 7]. Our approach is quite general since it utilizes only a consistent local ordering of topplings, following the instantaneous maximum gradient, and the notion of a metastable site. Furthermore, the model we propose shows a quasiperiodic time behavior, a feature already mentioned for SOC-related models in [8] and explored recently in a similar context by Chapman $[9]$.

The additional feature of the proposed model is the emergence of a spatial structure for the metastable configuration driven by the transport of grains through the system. A study of this spatial configuration in and close to the stationary state is the main objective of the fol-

\footnotetext{
*Electronic address: anton@ifin.nipne.ro
}

${ }^{\dagger}$ Electronic address: hbg@sun.ac.za lowing sections.

The paper is organized as follows. In Sec. II we describe the toppling rule and its connection with related models. In Sec. III we present the $1 \mathrm{D}$ variant of the model and in Sec. IV the results for the 2D version, followed by conclusions in Sec. V.

\section{THE TOPPLING RULE}

We consider a gradient-driven sandpile with a toppling rule which takes into account not only whether a local threshold gradient is exceeded, but also whether this situation is the result of the addition of a grain to the site under evaluation. We introduce this rule as a simple description of the dynamical weakening introduced in Ref. [5].

The general description of the dynamics is as follows. Grains are dropped on the sites in a designated region of the lattice called the source zone; following a relaxation rule, to be described in detail subsequently, grains can change their position on the lattice until they reach an open boundary and leave the system (lattice). In short, the model describes the transport of grains from the source zone to the open boundary.

Let us now analyze in detail the toppling rule in $2 \mathrm{D}$ on a square lattice. At a given moment of time each site of the lattice has an associated height $h$ of the grain column, and we also associate with it the set of gradients $G=\left\{g_{l}, g_{r}, g_{u}, g_{d}\right\}$, where $g_{\alpha}=h-h_{\alpha}$ and $h_{\alpha} \in\left\{h_{l}, h_{r}, h_{u}, h_{d}\right\}$ is the the height of the sand column at the four nearest neighbor sites left, right, up, and down.

We use two thresholds in our algorithm. (i) $g_{\max }$ is the stability threshold for the maximum of $G$. If at least one of the gradients of $G$ is larger than $g_{\max }$ the site is called metastable. It topples only if this state is the result of the receipt of a grain, either from a neighboring site which had toppled, or when a grain was dropped on the site at the start of an updating run. Metastable states do not topple when a gradient larger than $g_{\max }$ develops as a result of the loss of grains on neighbouring sites. (ii) $g_{\min }$ 
is the minimum positive gradient, which fixes the condition to stop the toppling; we call it the activity threshold. Once a site starts toppling, it sends grains, one at a time, along the instantaneous maximum gradient of $G$ to its nearest neighbors, provided that $\max \{G\}>g_{\min }$. If there is more than one instantaneous maximum gradient a random choice is made among them. If one site topples it will send grains to some of its nearest neighbors. We refer to the process of relaxation of one site as a "toppling" and to the sites that have received sand grains during toppling as "updated sites". Their coordinates are kept in a list for the next step of the dynamics.

In one time step all the updated sites produced at the previous time step are checked for stability and, if unstable, they relax according to the above algorithm. We specify that once a site is unstable and chosen to topple the algorithm will finish the toppling sequence at the site and then will move to another site. Physically, this is equivalent with neglecting the toppling time.

Because we always topple along the instantaneous maximum gradient we can introduce a time order of the topplings. On physical grounds it seems reasonable to assume that the site that first receives a grain will topple first at the next time step, if it is unstable. We model this using a first in, first out list: the coordinates of the updated sites are stored sequentially in a list. In one time step the algorithm reads the list sequentially from the first entry: if the current site is metastable, it is toppled and the sites that are updated in this process are stored in the list for the next time step; if the current site is stable, it is simply discarded from the list. We refer to this time ordered toppling rule by the acronym TOTR. (Recall that a gradient toppling rule implies a non-Abelian sandpile where the order of toppling has definite consequences; see, e.g., Refs. [3, 4].)

Alternatively, we can disregard the time ordering and select at random a site from the list of updated sites for inspection of its stability. In Sec. III we make a comparative study of these two variants of the toppling rules in the $2 \mathrm{D}$ case. We refer to this randomly ordered toppling rule by the acronym ROTR.

In other words, we can consider one time step of the dynamics, $\Delta t$, divided into $N$ bins ( $N$ being the number of sites of the lattice), each bin containing at most the coordinates of one updated site since in a time interval $\Delta t / N$ we can assume that typically at most one site is active. An active site generates a set of updated sites when topples. In this description the TOTR fills the bins of the next time step in the order in which the updated sites are produced at the current time step; meanwhile the ROTR fills the bins of the next time step randomly. Evidently, most of the bins are empty as the number of updated sites is much smaller than the system size and the algorithm discards the empty bins using the list of updated sites.

Thus, to resume, at a given instant of time we have three kinds of sites in our system: (i) inactive sites with all associated gradients less than $g_{\max }$; (ii) metastable sites, which have at least one associated gradient larger than $g_{\text {max }}$; and (iii) active or unstable sites, which are the toppling sites at the given instant (they are perturbed metastable sites).

One observation about the times scales in the model is now in order. We can think in terms of the existence of three time scales. The smallest one is the time in which a site has toppled, $\tau_{t}$. The second time scale is the surviving time of an updated unstable site, $\tau_{s}$. The dynamics of our model is such that $\tau_{s} \gg \tau_{t}$. The third time scale is the time between two droppings of grains on the lattice $\tau_{a}$. After one grain is dropped the system relaxes globally through an avalanche of topplings. Here we are ensured that $\tau_{a} \gg N_{L} \tau_{s}$, where $N_{L}$ is the average number of time steps for the system to relax for a given size $L$.

We define the size of an avalanche as the total number of topplings generated in a given relaxation process after one dropping.

We stress that our toppling rule allows and introduces sites with unstable gradients after one avalanche has taken place. They emerge as the neighbors of the toppling (active) sites toward which the gradient is negative. As an unstable site topples, the negative gradient increases in absolute value, but the sites along such a direction do not receive any grains and hence are not toppled, according to our rule. When topplings in such an avalanche stop, such a site can accordingly have a maximum gradient that is larger than the threshold value $g_{\max }$. We call such a site metastable - it can sustain gradients larger than the threshold as long as it remains unperturbed. Physically, we associate this rule with a certain local metastability of the medium through which transport takes place.

We make the observation that the metastable sites may be important in the regions where there is no dropping of grains, since they can live much longer than the average time between two droppings on the same site.

As elaborated in the next two sections, this model has properties similar to the extended version of the forest fire model described in Ref. [8], that is, quasiperiodic behavior in time and a spiked avalanche distribution.

To link further with previous studies, it is also of interest to understand the relation between our proposed model and the condition for SOC in sandpile models. References [8, 10] present an analysis to establish the generic conditions a model has to satisfy in order to present SOC. The authors show that in two dimensions, or larger, and for conservative dynamics, an intrinsic spatial anisotropy is required to produce SOC for models which can be treated perturbatively. The model we propose has a conservative toppling rule (the noise is also conservative in the region where no dropping takes place) and evolves in two spatial dimensions under conditions of anisotropy, but it does not present the features of SOC for the avalanche distribution.

We think that the explanation of this anomaly resides in the fact that the stationary state of this model cannot 
be characterised as a perturbation of an interaction-free model. As we present in full detail in Sec. IV] the stationary state of this model is characterized by the appearance of deep and narrow valleys along the direction of grain transport. These features cannot be obtained as a perturbation of a diffusionlike relaxation.

We also make the observation that in the proposed toppling rules there is no external noise term, e.g., similar to the noise term in the Langevin equation. The randomness in our model comes from the selection of the toppling order in the case of the ROTR, and from the random choice of the toppling direction in the case of a degenerate maximum gradient. Another source of randomness in these models is the 'internal noise', in the sense discussed in Ref. 11, coming from the fluctuations of the many particle dynamics which may be present even in the case of pure deterministic microscopic equations. There is no straightforward connexion between the 'internal noise' and the 'external noise' term of a Langevin equation.

In connection with this theoretical aspect we mention that recently a model with metastable states ("sticky grains" is the term used by the authors) but with a height-driven toppling rule and dissipative dynamics was studied in Ref. 12] and shown to be in the directed percolation universality class.

In the following sections we present a detailed discussion of the $1 \mathrm{D}$ case and a statistical analysis of the mentioned patterns of metastable states for the $2 \mathrm{D}$ case in the stationary state and close to the stationary state.

\section{THE 1D CASE}

We start our study with a one-dimensional sandpile. We choose a lattice of dimension $L$ with an open boundary at $x=L$ and with a wall at $x=0$. The grains are injected randomly in the region $x \in[1, w]$, with $1 \leq w \leq L$, called the source zone. We choose the stability threshold $g_{\max }=2$ and the activity threshold $g_{\min }=1$. Let us analyze in detail the appearance of a metastable site in 1D lattice. For simplicity we start with an initial stair configuration which has a monotonic step of $\max \{G\}=2$ descending from $x=1$ to $x=L$. This configuration is marginally stable; if we drop a grain anywhere on the lattice an avalanche occurs. First we consider a restricted source zone where grains are only dropped at $x=1$. A grain dropped at $x=1$ will then topple until $x=L$ for the chosen configuration. At $x=L$ an extra toppling takes place since $g_{\min }=1$, and the toppling of the disturbed state continues as long as the maximum gradient is larger than $g_{\text {min }}$. [Formally, the boundary condition is that $h(L+1)=0, h(0)=\infty$.] When the avalanche stops, we therefore see that the gradient between the sites $L-1$ and $L$ is 3 , hence we have a metastable site at $L-1$. Now if we drop a new grain at $x=1$, the same consideration shows that the metastable configuration moves to $L-2$. The process continues until the metastable configuration

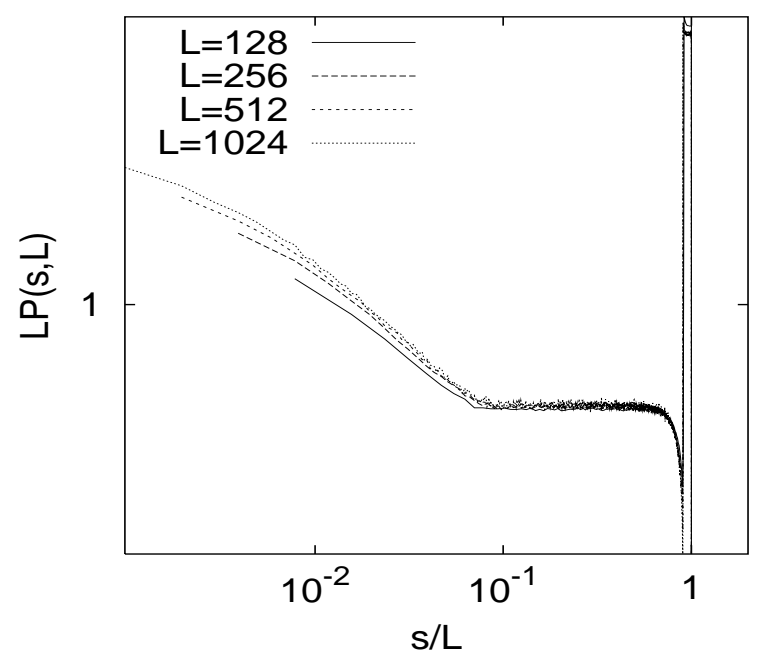

FIG. 1: The avalanche size distribution in $1 \mathrm{D}$ at $L=128$, 256,512 , and 1024 with the fixed ratio $w / L=0.1$. We notice the peak in the top right corner of the plot.

reaches the site $x=1$. After that a series of avalanches reconstructs the initial configuration, adding a grain at site $L$, then at $L-1$, and so on.

The characteristic time period of the system is controlled by the time in which the metastable site travels from the site $L$ to the site 1 . This kind of behavior is preserved if we use a source zone with $w>1$ but with the ratio $w / L$ small. The avalanche size distribution is characterized by a peak at the end of the distribution support (see Fig. 1), which results from the avalanches produced while the metastable site is present in the transport zone between $w+1$ and $L$. The smaller size avalanches are produced after the metastable site has reached the source zone. Figure 1 shows that for fixed ration $w / L$ the avalanche distribution scales with $L$.

We close this section with the observation that the distinction between the TOTR and ROTR is irrelevant in $1 \mathrm{D}$ since typically there is only one updated site in the algorithm.

\section{THE 2D CASE}

\section{A. General features}

In this section we compare the behavior of the system in two dimensions with the simple and well understood behavior in 1D. We choose for study a rectangular lattice of size $L$ with an open boundary condition at $x=L$ and a wall at $x=1$, with a periodic boundary condition along the $y$ axis. A simulation starts with the initial condition specified by a uniform slope of size 1 per lattice step 


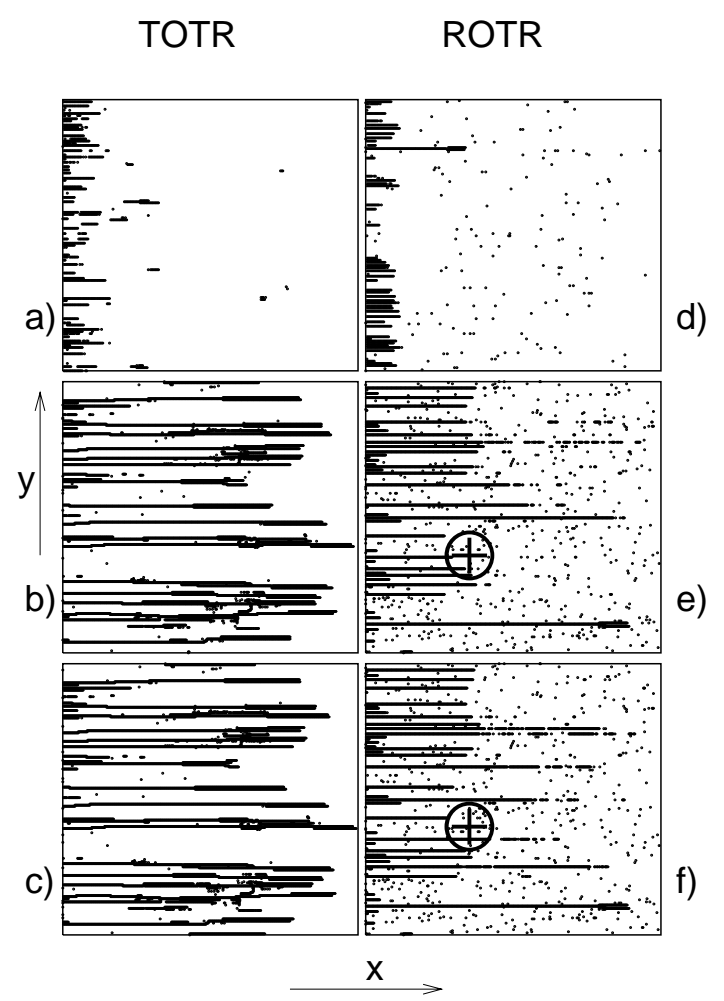

FIG. 2: The patterns created by sites at the bottom of valleys for a $512 \times 512$ lattice during the transient regime (a),(d) and in the stationary regime (b), (c), (e), (f). On the left side the dynamics is TOTR and on the right side the dynamics is ROTR. The dots, often merged to lines, mark the sites which have at least two negative gradients larger in absolute value than $g_{\max }$, i.e., at least two neighbors are metastable. (See the text for discussion of the symbols $\oplus$.)

along the $x$ axis, the height at $x=L$ being 0 . The initial slope along the $y$ axis is set to 0 . We choose the stability threshold $g_{\max }=4$ and the activity threshold $g_{\min }=1$. (We have also performed simulations starting with an empty lattice; the stationary state is the same and only the transient regime is longer.) We use both toppling rules: (i) with the time ordered toppling rule (TOTR) and (ii) with a random ordered toppling rule (ROTR).

The grains are dropped randomly at $x=1, y \in[1, L]$. This source configuration allows us to explore the behavior of the transport region of the sandpile under minimal perturbation, since only one grain topples at the boundary of the transport region and the metastable sites do not have their average lifetime constrained by the average time between two droppings on the same site; hence the structure of the transport zone is influenced only by a minimal flow of grains.

The initial condition we start with can be viewed as a collection of interacting 1D sandpiles oriented along the $x$ axis, and consequently one might expect to see two-dimensional avalanches created by the interaction of

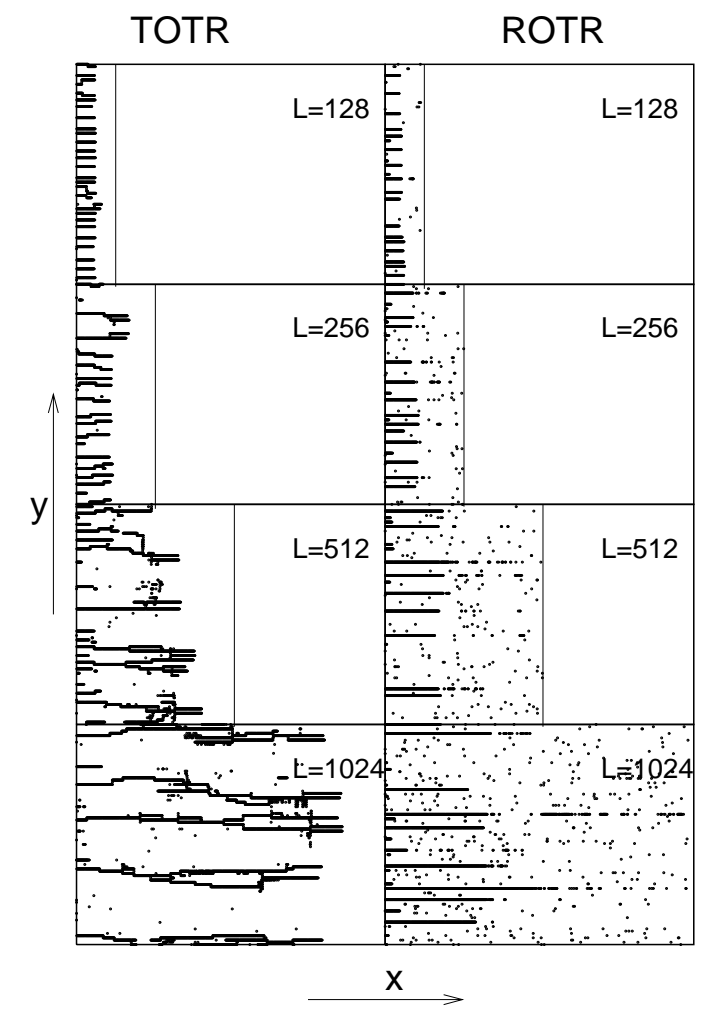

FIG. 3: The structure of the bottom of the valleys as a function of the lattice size $L$. From top to bottom $L=$ 128, 256, 512, 1024; TOTR on the left side and ROTR on the right side. The source is on the left side and the open boundary on the right side of each panel. The vertical lines mark the open boundary.

different metastable sites.

Surprisingly we found that in the stationary state the sandpile develops a structure of valleys along the $x$ direction separated by terraces of sites in the metastable state. As one can see from Fig. 22 the valleys are not purely 1D; instead they fluctuate slightly along the transverse direction and also show branched structures. Visual inspection of Fig. 2 shows that the transverse fluctuations and branching are more pronounced in the case of the TOTR algorithm.

After the system reaches the stationary state we notice that in the case of the TOTR the valleys do not change in time except for small fluctuations which appear toward the open boundary. In the case of the ROTR the valleys do change in time even in the stationary state. We illustrate this in Fig. 2 where the snapshots (c) and (f) follow after $10^{6}$ droppings on the snapshots (b) and (e) in the stationary regime. We observe that in the case of the TOTR the two configurations are almost identical, while for the ROTR there is a clear difference between the two configurations around the points denoted with the symbol $\oplus$. We return to this point when we analyze the cluster size distribution and the correlation length 


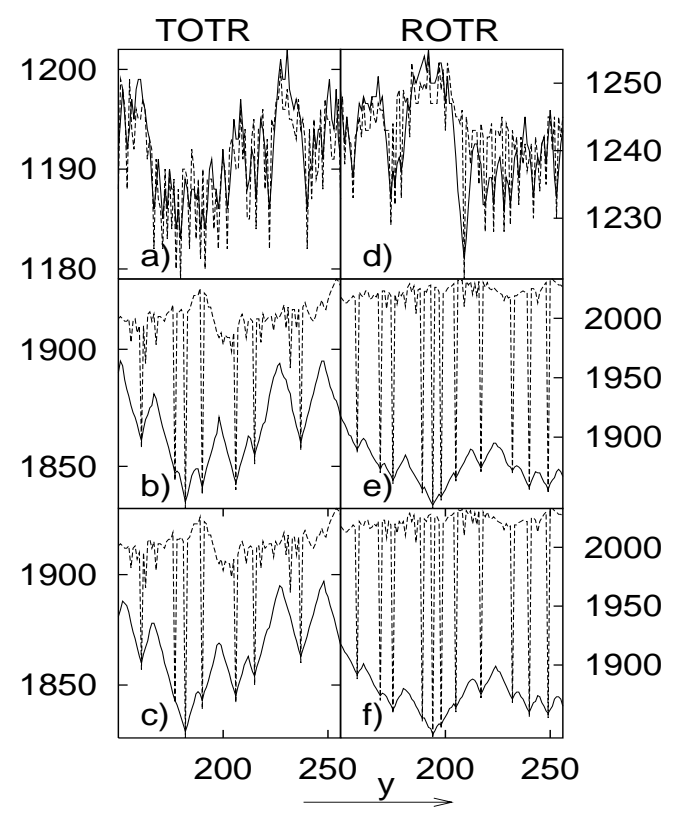

FIG. 4: The profile of the sandpile along the source line $x=1$ (continuous line) and along the line next to it, $x=2$ (dashed line), for TOTR (left) and ROTR (right). We show one moment in time of the transient regime. (a) and (d), while (b), (c) and (e), (f) are in the stationary regime. The coordinate is the height in number of grains. Note the change of the profile height with time, and the fact that the profile is unchanged in the stationary regime.

behavior.

Another observation which we can make from visual inspection of the stationary patterns, is that the structure of the valleys changes with system size. From Fig. 3 we see that for $L=128$ the valleys are predominantly onedimensional. When the lattice size increases, transverse fluctuation and branches appear.

We obtain further insight about the nature of the stationary state if we analyze the time evolution of the height profile along $z$ (perpendicular to the $x-y$ plane) at the boundary of the source zone. In Fig. [4 we show the time evolution of the height profile along $y$ for $x=1$ and $x=2$. We see that in the transient state, for both toppling rules, the source zone $(x=1)$ has heights close to the heights of the $x=2$ profile. In this configuration a grain dropped in the source zone will move more probably along the $x$ axis, leaving the source zone in one or a few steps. In contrast, in the stationary regime we see that a completely different configuration arises. The average height of the source zone is significantly smaller than the average height of the $x=2$ profile. A grain dropped in the source zone will therefore first travel along the $y$ direction, until it meets a minimum which is connected

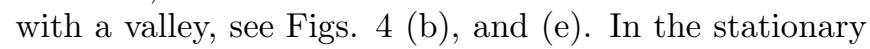
state the profile at the source remain unchanged for both

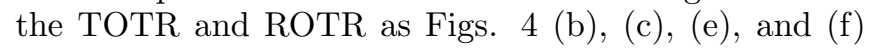

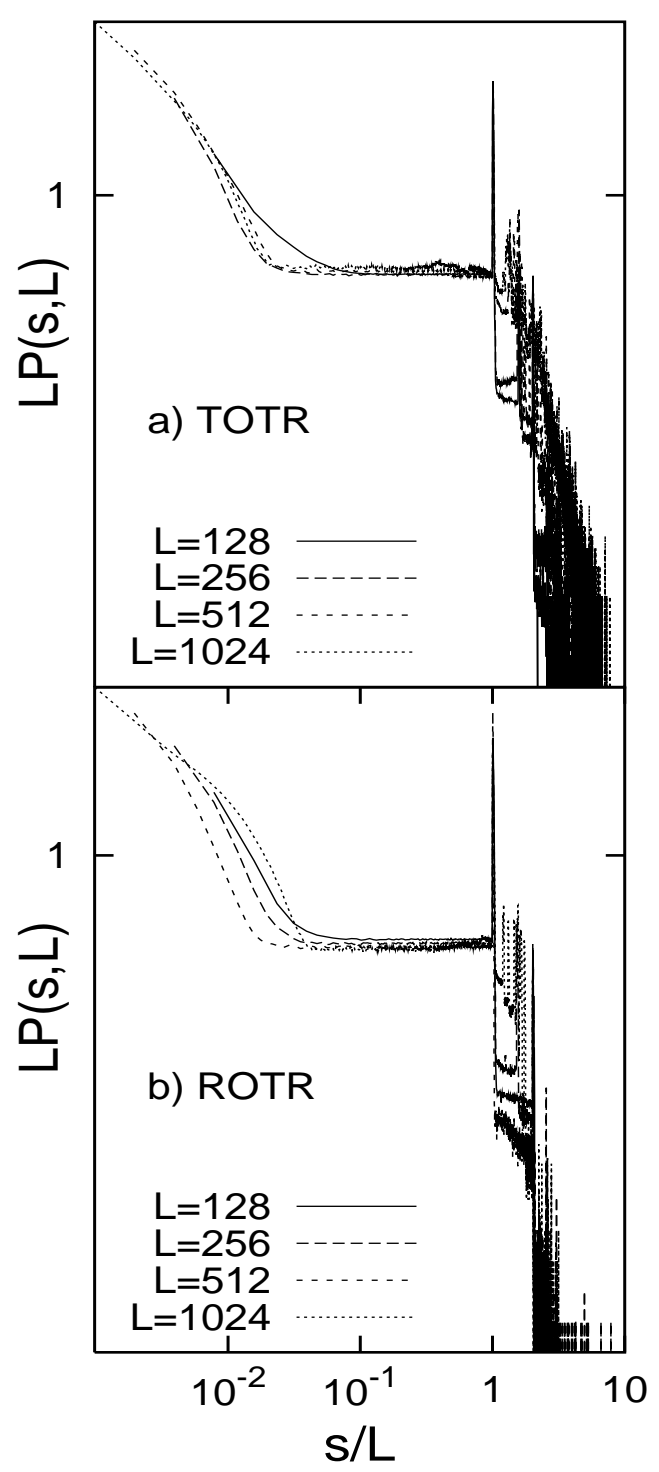

FIG. 5: The avalanche size distribution: (a) TOTR, (b) ROTR. The system size takes the values $L=$ $128,256,512,1024$. The plateau region and the first peak scale approximately with system size $L$.

show. The data are taken from the same configuration presented in Fig. 2 ]

We close the presentation of general features of the model with an analysis of the avalanche size distribution in the stationary state. Figure 5 shows for both dynamics that the size distribution is not power-law-like, but rather similar to the 1D case (compare Fig. 1), although with a richer structure of peaks. We remark that a scaling proportional with lattice size $L$ holds approximately for the plateau region and the first peak in the distribution, as 
in the 1D case. Since the valley length scales with $L$, we think that this feature of the avalanche size distribution is determined by the propagation of the avalanches along the valleys.

\section{B. Cluster characterization}

Having presented a visual description of the valleys in the previous subsection, we now consider a quantitative approach. For a numerical description of the patterns shown in Figs. 2 and 3 we concentrate on the clusters formed by the bottom of the valleys. We define a site to be at the bottom of a valley if it has associated with it two or more negative gradients larger in absolute value than the threshold, i.e., at least two neighbors are metastable.

A set of bottom of the valley sites is said to form a cluster if they can be spanned by a path stepping only to nearest neighbors along the $x$ or $y$ directions.

We analyze the following quantities: the total number of clusters $N_{c}(t)$ as a function of time, the total cluster mass $M_{c}(t)$ as a function of time, the longitudinal and transverse correlation lengths $\xi_{\|}$and $\xi_{\perp}$, and, finally, the cluster size distribution at a given time, $N(s, t)$.

We start with the time evolution of the total number of clusters $N_{c}(t)$ and the total mass $M_{c}(t)=\sum_{s} s N(s, t)$. We remind the reader that in this section a time step corresponds to a grain drop and the subsequent relaxation, and therefore we neglect the relaxation time per avalanche.

The data were collected over a single run starting with the initial condition specified at the beginning of this section. We have collected the data for one point at time intervals $5 \times 10^{4}, 10^{5}, 10^{6}$, and $5 \times 10^{6}$ for the system sizes $L=128,256,512,1024$, respectively. To eliminate the short time fluctuation we averaged at each point over a window of size $10^{4}$ steps from which we selected 100 moments equally spaced. We mention here that we are constrained to use single runs since the characteristic time in which the system reaches the stationary state scales approximately with the third power of the lattice linear size.

Figure 6 shows that the total mass of the clusters and the number of clusters have a jump before the stationary state is reached. The jump is more pronounced in the case of the TOTR and is clearer for large system size. We observe that the time to reach the stationary state scales with the third power of system size. This fact can be explained if we assume that the average slope of the system converges to a nonzero value as the system size is increased; then the volume of the accumulated grains scales like $L^{3}$, which determines the minimal number of time steps necessary to drive the system to the stationary state.

In studies of this kind of model it is customary to test the data for scaling behaviour $f(t, L)=L^{\alpha} \tilde{f}\left(t / L^{z}\right)$. Our plot shows that one can define a dynamical exponent $z=3$ for the relaxation time, but a simple proportional-

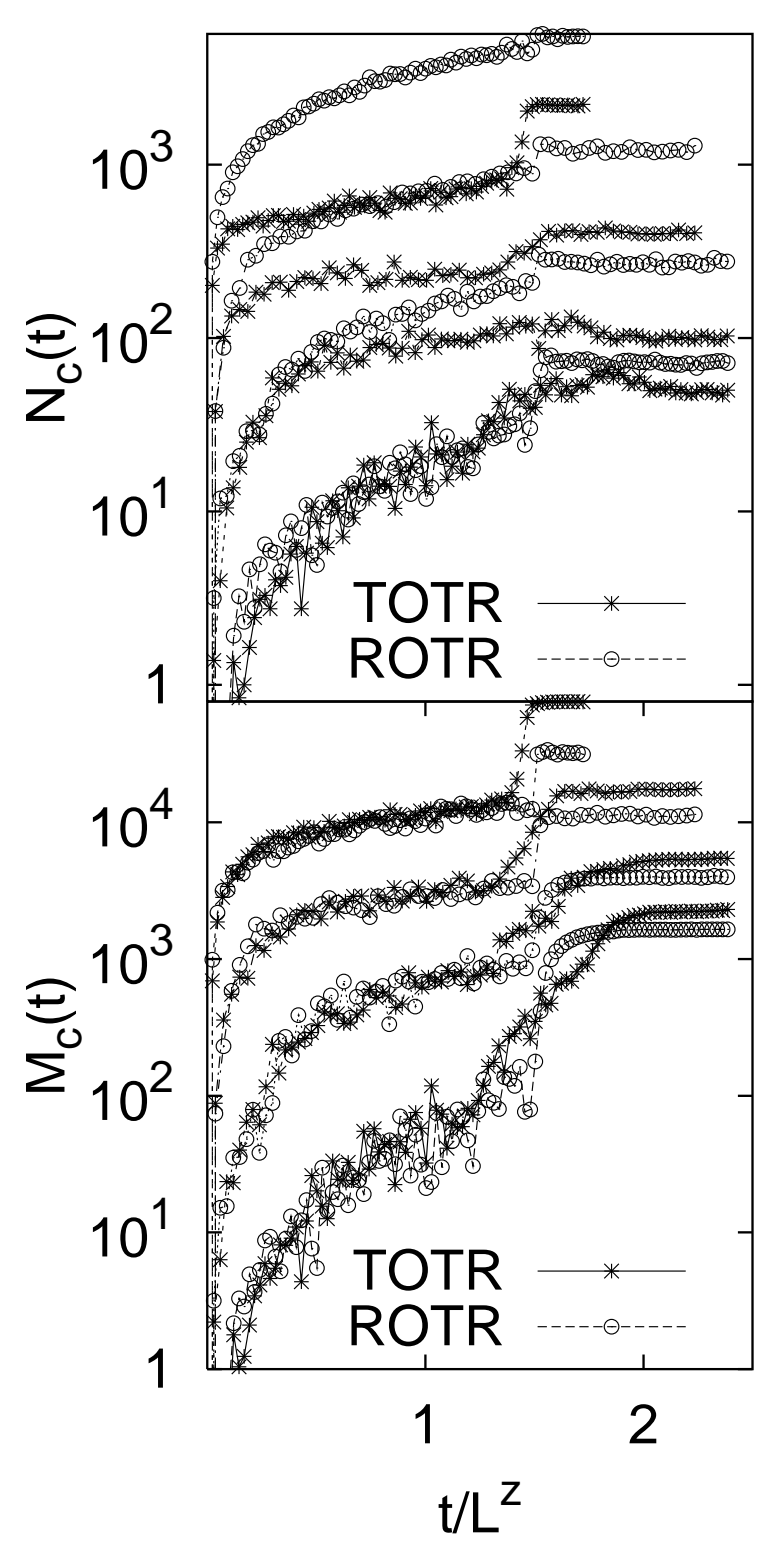

FIG. 6: Number of clusters (top) and their total mass (bottom) as function of time for the TOTR and ROTR. In ascending order the plots correspond to the lattice sizes $L=128,256,512,1024$.

ity with the system size at a given power is not sustainable, although for $L=512,1024$ the assumption holds acceptably (the corresponding lines are parallel within a good approximation). However, the scaling hypothesis does not hold for the system size $L=128$, suggesting that boundary effects have a characteristic length of this order of magnitude. From Fig. 3 we see that close to the the source zone the valleys do not present branching. We note also that close to the open boundary valleys do not form, which makes it plausible that the scaling is strongly affected by finite size effects.

For a geometric charaterization of the clusters we define the following correlation lengths, following Ref. [13]: 


\section{TOTR ROTR}

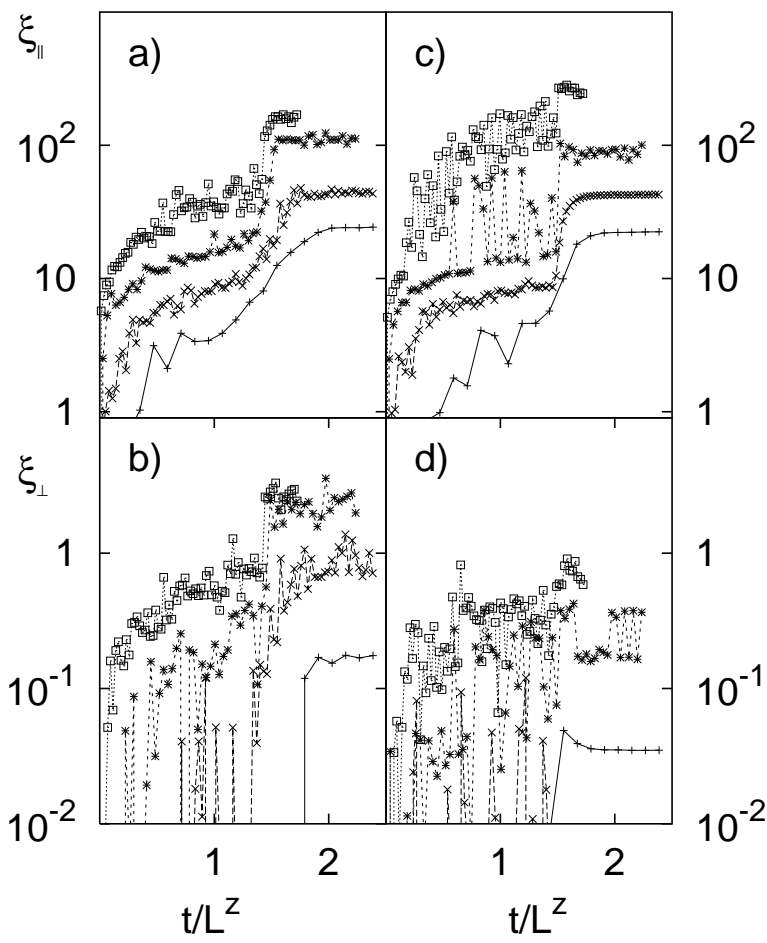

FIG. 7: Correlation lengths $\xi_{\|}$(top) and $\xi_{\perp}$ (bottom) as function of time [ $\mathrm{L}=128(+), 256(\times), 512(*), 1024(\square)]$. Left panels are for TOTR right panels for ROTR.

$$
\xi_{i}^{2}=\frac{2 \sum_{s} R_{i, s}^{2} s^{2} n_{s}}{\sum_{s} s^{2} n_{s}}
$$

where $R_{i, s}^{2}$ is the mean square distance along the longitudinal, $x$, or transverse, $y$, direction for the sites belonging to one cluster averaged over all clusters of size $s$, which can be expressed in the formula

$$
2 R_{i, s}^{2}=\frac{1}{n_{s}} \sum_{\alpha=1}^{n_{s}} \frac{1}{s^{2}} \sum_{m_{\alpha}, n_{\alpha}}\left|x_{m_{\alpha}}^{(i)}-x_{n_{\alpha}}^{(i)}\right|^{2} .
$$

Here the index $i=\|, \perp$ indicates the longitudinal and transverse directions, $x_{n_{\alpha}}^{(i)}$ denotes the coordinate of the sites belonging to the $\alpha$ th cluster of size $s$, and $n_{s}$ is the number of clusters with size $s$. In other words, for each cluster from a given configuration of the lattice we compute its average square displacement from the center of mass in transverse and longitudinal directions with Eq. (2). Next we average over all the clusters from a configuration with weights specified by Eq. (1).

Figure [7] shows that the longitudinal correlation lengths jump before the stationary regime for system sizes $L=512$ and 1024 , similar to the behavior of the cluster number and the total mass( see Fig. 6).

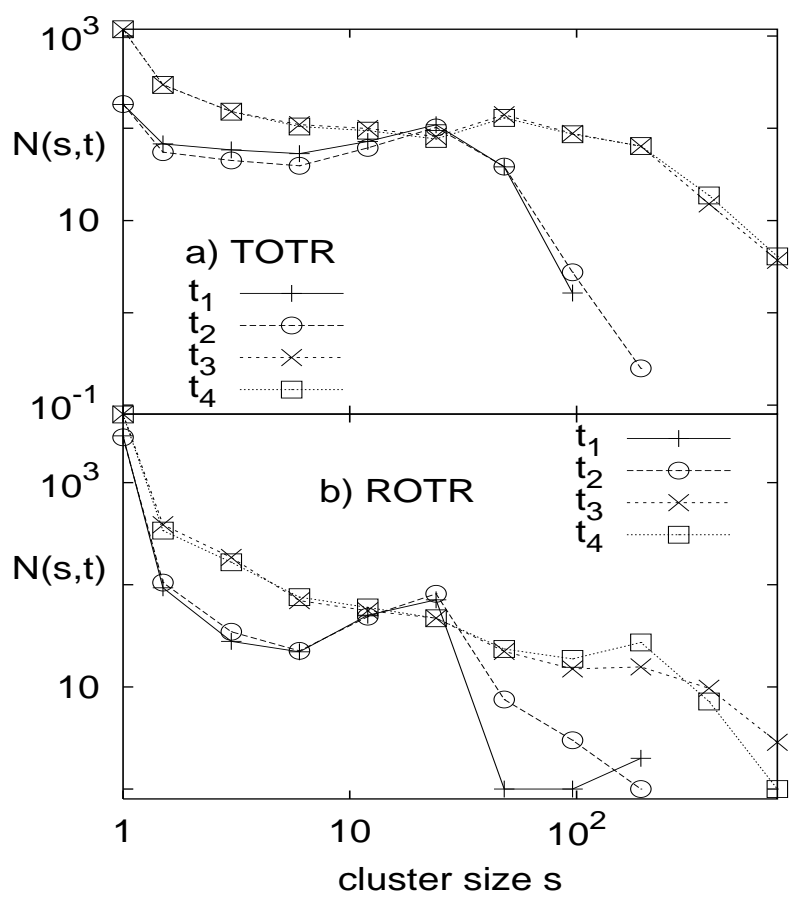

FIG. 8: Histogram of cluster sizes at four moments of time. We take $t_{1}$ and $t_{2}$ in the transient regime with $\xi_{\|}\left(t_{1}\right)<$ $\xi_{\|}\left(t_{2}\right)$ and $t_{3}, t_{4}$ in the stationary regime with $\xi_{\|}\left(t_{3}\right)<$ $\xi_{\|}\left(t_{4}\right)$. We mention that data are averaged around each time moment $t_{i}, i \in[1,4]$, as described at the beginning of Sec. IV B.

It is intriguing that the longitudinal correlation length shows chaotic behavior in the transient state as the system size increases, especially in the case of the ROTR, even though we have averaged out the short time fluctuations. On the other hand, the time evolution of the total number of clusters does not present fluctuations of the same relative magnitude (see Fig. 6). We conjecture that the large fluctuation of the correlation lengths in the case of the ROTR is related to a high frequency of coalescence and breaking up of clusters before the stationary state is reached.

The transverse correlation presents the same kind of chaotic behavior. In any event, we see that $\xi_{\perp} \geq 1$ (which is physically significant) only for the TOTR with $L \geq$ 512. Visual inspection of Figs. 2 and 3 shows that it is this situation in which a significant number of branchings are observable.

To elucidate this point further, we study, for both types of dynamics TOTR and ROTR, the cluster size distribution at various moments of time for the lattice size $L=1024$. In Fig. 8 we plot the cluster distribution at two consecutive moments in the transient 
regime $\left(t_{1}=8.7 \times 10^{8}, t_{2}=8.75 \times 10^{8}\right)$ corresponding to a minimum and a maximum of $\xi_{\|}$observed in Fig. 7 that is, $\xi_{\|}\left(t_{1}\right)<\xi_{\|}\left(t_{2}\right)$, and in the stationary state $\left(t_{3}=1.84 \times 10^{9}, t_{4}=1.845 \times 10^{9}\right)$ with the same condition $\xi_{\|}\left(t_{3}\right)<\xi_{\|}\left(t_{4}\right)$.

The plots show that in the transient regime the states with larger $\xi_{\|}$have a significant population of large clusters for both dynamics. In the stationary state in the case of the TOTR the cluster distribution changes very little, while for the ROTR the the large size tail presents observable fluctuations. This observation seems to point to a signature for a stationary state of the ROTR in which large scale fluctuations appear a the cluster of valleys, as shown in Fig. 2] (We have also checked this fact for other configurations.)

To summarize the two-dimensional behavior, we have studied the geometric and relaxation properties of the transport region of the sandpile. We found that as the system approaches the stationary state, clusters of deep valleys appear. Specifically, we explored the time evolution for the total number of clusters, the total mass of the clusters, the correlation lengths (longitudinal and transverse) of the clusters, and the cluster size distribution at various moments of time. The main distinction between the TOTR and ROTR is the fact that the configurations obtained from the ROTR are strongly affected by fluctuations of the longitudinal correlation length, which we have shown to be associated with fluctuation in the tail of the cluster size distribution.

The characteristic time to reach the stationary state scales with the system size as $L^{z}$, with $z \approx 3$, but the magnitude of the observed quantities does not follow a clear power law type of scaling. The range of data we have does not allow us to decide if this is due to strong correction from boundary effects, or if scaling is genuinely broken. The setting in of a chaotic regime at large $L$ complicates the analysis even further.

\section{CONCLUSIONS}

We have analyzed a gradient-driven sandpile model with local metastable states using two variants of the toppling rule: one which keeps the time order of the list of updated sites and one which selects a site from the list at random. We found that in $2 \mathrm{D}$ metastable sites generate a rough landscape with deep valleys along which grains are transported.

The valleys are organized in clusters with a pronounced development along the direction of grain flow $\left(\xi_{\|} \gg \xi_{\perp}\right)$. For the time ordered toppling rule, we found that transverse correlations develop as the system size increases. Also, for large system size we observed chaotic behavior for the correlation lengths, associated with fluctuations in the size distribution of the clusters.

The avalanches produced in the resulting valleys have one-dimensional properties and show non-SOC behavior. We believe that this is an example of a model in the strong coupling regime which escapes the classification made in Ref. 10] for models that can be treated perturbatively. Yet we do not have an exact mapping between our discrete model and the continuum Langevin equations used in Ref. 10]. This may be another source of the discrepancy. At the moment this is a purely numerical study of a descriptive nature. To clarify the previous uncertainties further analytical insight seems to be required. It would, e.g., be interesting to deduce a simple mean field kind of equation capable of explaining the irregular time behavior at large $L$ for the quantities described in the paper.

\section{Acknowledgments}

The authors thank an anonymous referee for detailed comments and useful suggestions. One of the authors, L A, thankfully acknowledges the hospitality of the Department of Physics, Inha University, South Korea, where part of the work was done; L. A. also acknowledges partial support from the Marie Curie Fellowship of the European Community programme under Contract No. HPMF-CT-2002-01910 . We acknowledge early discussions with Morne Pistorius and preliminary calculations by him which stimulated the present investigation.
[1] P. Bak, C. Tang, and K. Wiesenfeld, Phys. Rev. Lett. 59, 381 (1987).

[2] A. Mehta, J. M. Luck, and R. J. Needs, Phys. Rev. E 53, 92 (1996).

[3] H. Jensen, Self-Organized Criticality (Cambridge University Press, Cambridge, U.K., 1998).

[4] D. Dhar, Physica A 263, 4 (1999).

[5] K. Dahmen, D. Ertaş, and Y. Ben-Zion, Phys. Rev. E 58, 1494 (1998).

[6] P. Ball, The Self-Made Tapestry (Oxford University Press, Oxford, U.K., 1999).

[7] T. Shinbrot and F. Muzzio, Nature 410, 251 (2001).
[8] J. E. S. Socolar, G. Grinstein, and C. Jayaprakash, Phys. Rev. E 47, 2366 (1993).

[9] S. C. Chapman, Phys. Rev. E 62, 1905 (2000).

[10] G. Grinstein, D.-H. Lee, and S. Sachdev, Phys. Rev. Lett. 64, 1927 (1990).

[11] N. G. Van Kampen, Stochastic processes in physics and chemistry (Elsevier, Amsterdam, 1992).

[12] P. K. Mohanty and D. Dhar, Phys. Rev. Lett. 89, 104303 (2002).

[13] D. Stauffer and A. Aharony, Introduction to Percolation Theory (Taylor \& Francis, London, 1992). 